\section{Assessment of Efficacy of Dexmedetomidine and Fentanyl in Combination with Propofol for Ease of Laryngeal Mask Airway Insertion and Haemodynamic Stability}

KEY WORDS: Laryngeal mask airway, Anesthesia, Dexmedetomidine, Fentanyl, Propofol, Haemodynamic, Bradycardia
Dr Kankudte Akshay O* Dr Sunil S Lawhale
Junior Resident In Department Of Anaesthesiology At Dr PDMMC Amravati. *Corresponding Author
HOD In Department Of Anaesthesiology At Dr PDMMC Amravati

Background: Laryngeal mask airway (LMA) insertion requires anesthesia and suppression of airway reflexes. In search of an optimal drug, we compared dexmedetomidine and fentanyl, in combination with Propofol for ease of LMA insertion and haemodynamic stability. Method: Total 120 patients belonging to ASA status I \& II posted for elective surgery were randomly divided into 2 equal groups. Both the groups received I.V glycopyrrolate $4 \mu \mathrm{g} / \mathrm{kg}$ prior to receiving the study drugs. Group D received dexmedetomidine I.V $1 \mu \mathrm{g} / \mathrm{kg}$ diluted in 10cc NS over 10min while group F received fentanyl I.V $2 \mu \mathrm{g} / \mathrm{kg}$ diluted in $10 \mathrm{cc}$ NS over $10 \mathrm{~min}$. Induction was done with IV Propofol $2 \mathrm{mg} / \mathrm{kg}$ in both the groups. After $90 \mathrm{sec}$, LMA insertion (no 3 for females and no. 4 for males) was done by the consultant anaesthesiologist blinded to the technique. Results: Jaw opening, ease of LMA insertion, requirement of additional Propofol was clinically insignificant and comparable between two groups. Cough though seen more in fentanyl group, $(p=0.042)$ while the incidence of bradycardia was more with dexmedetomidine group. Between the two groups, the change in blood pressure from baseline to $30 \mathrm{sec}$ after induction and upto $10 \mathrm{~min}$ after LMA insertion was statistically insignificant. Within the individual groups, the changes in the blood pressure reached statistical significance. These changes did not reach a clinical significance and required no additional medication. Conclusion: Either dexmedetomidine or fentanyl when used along with propofol provides comparable conditions for the ease of LMA insertion with stable haemodynamic parameters in pre-hydrated, healthy and young patients.

\section{INTRODUCTION}

Supraglottic airway devices have become a standard fixture in airway management, filling a niche between the facemask and tracheal tube in terms of both anatomical position and degree of invasiveness [1]. Laryngeal mask airway (LMA) is a non-invasive supraglottic device which has the advantage of being less stimulating than tracheal intubation as visualization of cords and introduction into trachea is not required [2]. Inadequate depth of anaesthesia may provoke coughing, gagging and laryngospasm which may lead to adverse haemodynamic changes and increased incidence of aspiration and regurgitation $[3,4]$. Therefore LMA insertion requires an optimal depth of anaesthesia. Propofol when used alone requires a higher dose to achieve adequate depth for LMA insertion. A dose more than $2.5 \mathrm{mg} / \mathrm{kg}$ causes cardiorespiratory depression. Other problems associated with it include apnoea, hypotension, excessive patient movement and laryngospasm. So Propofol as a single agent is unsatisfactory and to overcome problems associated with it, a number of other adjuvant agents have been introduced [5].

The addition of fentanyl has been shown to improve the insertion conditions with an overall success rate of up to 8595\% [6]. In our institute, it is a practice to use fentanylmidazolam as a premedication and Propofol as an induction agent for LMA insertion. Unfortunately these medications also increase the incidence and duration of apnoea [7].

In order to decrease the adverse effects of propofol, fentanyl and now newera2-agonists such as dexmedetomidine or muscle relaxants were added to reduce the propofol dose requirement. It has analgesic and sedative effects with little effect on ventilation [8] and also used as an adjuvant in general anaesthesia and for postoperative sedation in the ICU for less than 24 hours [9, 10]. Anxiolysis, blood pressure stabilisation, analgesia, anaesthetic sparing effects and sedation without respiratory depression or significant cognitive impairment effects of dexmedetomidine are known [11].These properties of dexmedetomidine make this drug of particular interest for premedication. However, it has the ability to reduce the analgesic requirements of opioids as well as the requirement of volatile and regional agents. In present study we have done a comparison between dexmedetomidine and fentanyl with Propofol as an induction agent for assessing the haemodynamic stability and laryngeal mask airway insertion conditions.

\section{MATERIALS AND METHODS}

This randomized double blinded study was conducted in total 120 patients of ASA status I \& II, age between 18-65 years, $\mathrm{BMI} \leq 30$ and posted for elective surgery under general anaesthesia with LMA during a period from lst Jan 2018- 30 June 2019. Patients with ASA physical status III \& IV, cardiovascular, respiratory, metabolic, endocrine disease, moderate to severe renal or hepatic impairment, allergy to any of the study drugs, restricted mouth opening, BMI $>30$ and reactive airway disease, gastro-oesophageal reflux, hiatus hernia, history of drug and alcohol abuse, patient refusal, pregnant and lactating women were excluded from the study. Preoperatively, complete blood count, blood urea nitrogen, serum creatinine, random blood sugar and chest X-ray PA view were performed for every patient. 12 lead ECG was done for patients above 35years of age. Since our study included more number of day care surgeries, all patients' in the study were not premedicated. All were kept fasting for six hours prior to surgery. After explaining the protocol, a written informed consent was obtained from all the patients. After attaching the monitors, baseline readings were recorded. Then intravenous access was secured and Ringer's lactate infusion was started. The patients were preoxygenated with $100 \%$ oxygen via a facemask for 3 minutes before induction of general anaesthesia.

Random sampling with patient allocated to even and odd group randomly. Both the groups received I.V glycopyrrolate $4 \mu \mathrm{g} / \mathrm{kg}$ prior to receiving the study drugs. Patients belonging to group D received injection dexmedetomidine I.V $1 \mu \mathrm{g} / \mathrm{kg}$ diluted in 10cc NS over 10min. Whereas patients belonging to group F received injection fentanyl I.V $2 \mu \mathrm{g} / \mathrm{kg}$ diluted in $10 \mathrm{cc}$ NS over 10min. After the study drugs were administered induction was done with I.V Propofol $2 \mathrm{mg} / \mathrm{kg}$ in both the groups. After $90 \mathrm{sec}$, LMA insertion (no 3 for females and no. 4 for males) was done by the consultant anaesthesiologist blinded to the technique described by Brain [12]. If LMA insertion failed due to inadequate relaxation; $0.5 \mathrm{mg} / \mathrm{kg}$ of additional Propofol was injected. Insertion conditions such as Jaw opening, Number of attempts, Ease of LMA insertion, unwanted responses like gagging, coughing, laryngospasm, additional requirement of Propofol during LMA insertion and abnormal movements like myoclonus and other involuntary 
movements) were assessed. After successful LMA insertion, anaesthesia was maintained with $1.5 \%$ sevoflurane and $50 \%$ nitrous oxide in oxygen using a circle absorber system. Adverse effects were assessed in both the groups and haemodynamic parameters (Heart rate (HR), Systolic BP (SBP), Diastolic BP (DBP), Mean arterial pressure (MAP), Respiratory Rate (RR) and SpO2) were measured at baseline, before LMA insertion (30sec, $1 \mathrm{~min}$ ) and up to 10 minutes (2min, 3min, $5 \mathrm{~min}, 7 \mathrm{~min}, 10 \mathrm{~min}$ ) after LMA insertion. Cardiovascular interventions included IV ephedrine $6 \mathrm{mg}$ if the SBP decreased to $80 \mathrm{mmHg}$ and IV atropine $0.6 \mathrm{mg}$ if HR decreased to less than $45 \mathrm{bpm}$.

\section{Statistical Analysis}

Patient data was summarized as mean and standard deviations (SD). Comparison between two groups with respect to continuous variables such as age, weight, $H R$, SBP, DBP, MAP and apnoea time was compared with student's t-test. Parameters measured over multiple points of time were analyzed using repeated measures ANOVA with Bonfernii post-hoc test. Categorical variables like gender distribution were compared by Chi-square test. All statistical analysis was done by using Statistical Packages for Social Sciences (SPSS) version 17.0. A p-value of $<0.05$ was considered as statistically significant.

\section{OBSERVATIONS AND RESULTS}

A total of 120 patients belonging to ASA status I \& II were enrolled and randomized into two groups of 60 patients in each. The demographic data of the patients were comparable and found no significant difference between two groups with respect to age, sex distribution, BMI, ASA status as shown in table 1.

Table 1: Demographic profile of the patients

\begin{tabular}{|c|c|c|c|c|}
\hline \multicolumn{2}{|c|}{ Parameters } & Group F & Group D & P value \\
\hline \multicolumn{2}{|c|}{ Age (in years) } & $42.80 \pm 12.32$ & $40.06 \pm 10.66$ & 0.196 \\
\hline \multicolumn{2}{|c|}{ BMI } & $24.99 \pm 1.50$ & $25.11 \pm 1.54$ & 0.667 \\
\hline \multirow{2}{*}{ Gender } & Male & $38(63.3 \%)$ & $34(56.7 \%)$ & 0.576 \\
\cline { 2 - 4 } & Female & $22(36.7 \%)$ & $26(43.3 \%)$ & \\
\hline \multirow{2}{*}{ ASA } & I & $36(60 \%)$ & $40(66.7 \%)$ & \multirow{2}{*}{0.570} \\
\cline { 2 - 4 } & II & $24(40 \%)$ & $20(33.3 \%)$ & \\
\hline
\end{tabular}

Jaw opening, attempts for LMA insertion, ease of LMA insertion and requirement of additional propofol was clinically insignificant and comparable between both the groups. Adverse reactions like laryngospasm and vocalization were not seen in both the groups. Cough though seen more in fentanyl group and was clinically significant $(p=0.042)$. In both group 4/60 (3.3\%) patients' shows gag reflex having $p$ value of 1.000 which was not significant. In $F$ group $4 / 60(6.6 \%)$ patients shows involuntary movements where as in group D 6/60 (10.0\%) patients shows involuntary movements $(\mathrm{P}=0.509)$ which was statistically insignificant, (Table 2).

Table 2: Comparison of LMA insertion conditions between two groups

\begin{tabular}{|c|c|c|c|c|}
\hline \multicolumn{2}{|c|}{ Parameters } & Group F & Group D & P value \\
\hline Jaw opening & Full & $54(90 \%)$ & $56(93.4 \%)$ & 0.509 \\
\cline { 2 - 4 } & Partial & $6(10.0 \%)$ & $4(6.6 \%)$ & \\
\hline \multirow{2}{*}{$\begin{array}{c}\text { Attempts for LMA } \\
\text { insertion }\end{array}$} & I & $56(93.4 \%)$ & $56(93.4 \%)$ & 1.000 \\
\cline { 2 - 4 } $\begin{array}{c}\text { Ease of LMA } \\
\text { insertion }\end{array}$ & II & $04(6.6 \%)$ & $04(6.6 \%)$ & \\
\cline { 2 - 4 } Cough & Easy & $56(9.6 \%)$ & $04(6.6 \%)$ & 1.000 \\
\hline \multirow{2}{*}{ Gag } & No & $56(93.4 \%)$ & $60(93.4 \%)$ & \\
\cline { 2 - 4 } & Yes & $04(6.6 \%)$ & $0(0.0 \%)$ & \\
\hline \multirow{2}{*}{$\begin{array}{c}\text { Involuntary } \\
\text { Movements }\end{array}$} & No & $56(93.4 \%)$ & $56(93.4 \%)$ & \multirow{2}{*}{1.000} \\
\cline { 2 - 4 } & Yes & $04(6.6 \%)$ & $04(6.6 \%)$ & \\
\cline { 2 - 4 } & hand & $4(6.6 \%)$ & $6(10 \%)$ & 0.509 \\
\hline None & $56(93.4 \%)$ & $54(90.0 \%)$ & \\
\hline Laryngospasm & No & $60(100 \%)$ & $60(100 \%)$ & - \\
\hline
\end{tabular}

\begin{tabular}{|l|l|l|l|l|}
\hline Additional requirement & No & $54(90 \%)$ & $56(93.4 \%)$ & 0.280 \\
\cline { 2 - 5 }
\end{tabular} of Propofol \begin{tabular}{|l|l|l|}
\hline Yes & $06(10.0 \%)$ & $04(6.6 \%)$ \\
\hline
\end{tabular}

There was fall in heart rate in $\mathrm{D}$ group of patients as compared to F group of patients but not at medical significant level for which medication in the form of atropine is required. Comparison between the two groups did not show a significant difference in the systolic BP, DBP and MAP at baseline and various time intervals after administration of the study drug as depicted in figure 1 .

Figure 1: Comparison of haemodynamic parameters between two groups

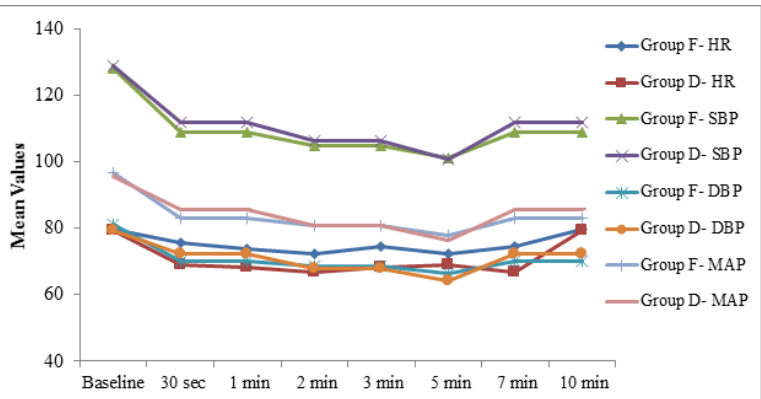

Time Intervals

Comparison between the two groups show a significant difference in the mean $R R$ at $30 \sec (p=0.034), 1$ min $(p=0.034)$ and at $2 \min (p=0.034)$ after administration of the study drug. There was fall in RR in F group of patients as compared to D group of patients as fentanyl is known as respiratory depressant drug, (Figure 2 ).

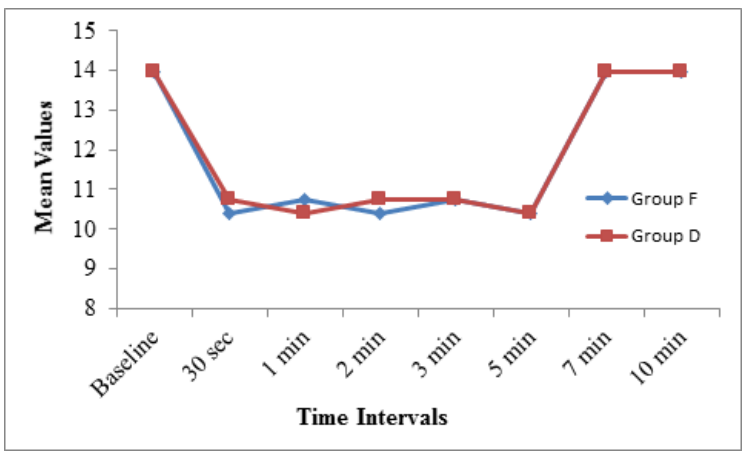

Figure 2: Comparison of respiratory rate between two groups

SpO2 was measured at Baseline, 30sec, lmin, 2min, 3min, $5 \mathrm{~min}, 7 \mathrm{~min}, 10 \mathrm{~min}$ interval and there was found to be statistical significance difference within both group (both Group F \&Group D) with $(p<0.001)$. Also between the groups (Group F \& Group D) there was no statistical significant difference from baseline to $10 \mathrm{~min}$ after LMA insertion, (Figure 3).

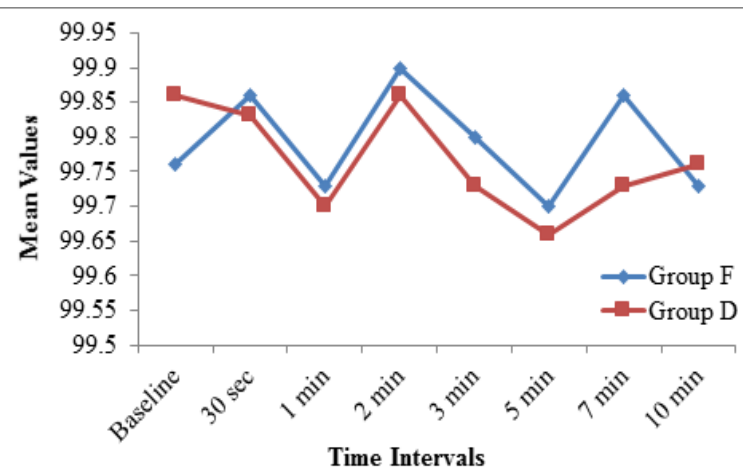

Figure 3: Comparison of SPO2 between two groups |www.worldwidejournals.com 


\section{DISCUSSION}

The introduction of LMA by Brain in the late 1980s has been one of the significant advances in the anaesthetic practice in the recent years. When propofol is used along with either fentanyl or dexmedetomidine, it provides stable cardiorespiratory condition, diminished airway reflexes and smooth insertion of LMA. In the present study LMA insertion conditions were assessed using an unvalidated methodology based on a 6 variable, 3 grade score proposed by Cheam et al [6] and Sivalingham et al [13]. In group D, 93.4\% patients as compared to $90 \%$ patients in group $\mathrm{F}$ had full jaw relaxation. Ease of LMA insertion was equal in both the groups. Vocalization and laryngospasm were not seen in any of the study groups. The number of attempts required for LMA insertion was also equal in both the groups. Thus it was concluded that both dexmedetomidine and fentanyl provided comparable LMA insertion conditions. These findings are correlated with the study done by Uzumcugil et al [14] and Hanci et al [15], in both the study dexmedetomidine is as successful as fentanyl in terms of mask placement conditions.

In current study, the incidence of cough was $6.6 \%$ in group F while none of the patients in group $D$ had incidence of coughing. This however reaches a statistical significance. In a study by Hanci et al [15] diaphragmatic movements and coughing in response to intubation and inflation of the ETT were significantly lower in dexmedetomidine group than in fentanyl group $(p<0.039)$. We used $2 \mathrm{mg} / \mathrm{kg}$ Propofol for induction. When there was a failure to insert LMA in the first attempt, $25 \%$ additional dose was administered. Only $6.6 \%$ patients in group D required $25 \%$ of additional Propofol while $10 \%$ of patients in group F required additional Propofol. This was however statistically not significant. The dose of $2 \mathrm{mg} / \mathrm{kg}$ Propofol was adequate for LMA insertion. Uzumcugil et al [14] used $1.5 \mathrm{mg} / \mathrm{kg}$ of Propofol for induction and found this dose suitable for LMA insertion when used with fentanyl $(1 \mu \mathrm{g} / \mathrm{kg})$ or dexmedetomidine $(\mathrm{l} \mu \mathrm{g} / \mathrm{kg})$.

Haemodynamic stability was also comparable between the two groups although the incidence of bradycardia was more with dexmedetomidine group. Between the two groups, the change in blood pressure from baseline to 30 sec after induction and upto 10min after LMA insertion was statistically insignificant. Within the individual groups, the changes in the blood pressure reached statistical significance. These changes did not reach a clinical significance and required no additional medication in the form of atropine was administered. It should however be noted that all patients were administered with IV glycopyrrolate $4 \mu \mathrm{g} / \mathrm{kg}$ before administering the study drugs. In spite of the statistically significant changes within the groups, there was no additional requirement of ephedrine in order to maintain haemodynamic stability. The change in Oxygen Saturation (SpO2) within the group was statistically significant at the entire interval $(p<0.001)$. But when it was compared between the two groups (Group F \& Group D) it was statistically insignificant. The change in $R R$ within the group was statistically significant at all the study interval $(p<0.001)$. But when compared between the groups (Group F \& Group D) it was statistically significant at $30 \mathrm{sec}, 1 \mathrm{~min}, 2 \mathrm{~min}$ interval after the induction and insertion of LMA $(p=0.034)$. These findings are comparable with the previous studies [16-19].

Present study did not require additional medication in the form of atropine. This could be attributed to the administration of IV glycopyrrolate prior to administering the study drug. One case which was to be included in the study was postponed since I.V dexmedetomidine when administered caused sudden increase in the blood pressure along with bradycardia. The blood pressure remained on the higher side for $30 \mathrm{~min}$. The patient belonged to ASA status I and was posted for operative hemorrhoidectomy. The blood pressure remained on the higher side in spite of giving her a benzodiazepine and an opioid. It has been observed that following a bolus dose of dexmedetomidine, an increase in blood pressure of $22 \%$ and a decrease in HR of $27 \%$ may occur due to its effects on peripheral $\mu 2$ adrenoceptors. Severe bradycardia following administration of $\mu 2$ agonists is well documented. Similarly Erkola et al [20] found a significant increase in the incidence of bradycardia in patients who had received intramuscular dexmedetomidine.

As a limitation to our study, we did not include the apnoea time. Also measuring RR rate shows observer variation from individual. We carried out controlled ventilation using $50 \%$ nitrous oxide, oxygen and $1.5 \%$ sevoflurane following LMA insertion, hence could not assess these parameters as a comparison between the two groups. The study was only limited to $10 \mathrm{~min}$ following LMA insertion and hence we did not assess the intraoperative haemodynamics and additional need for opioid or a relaxant if required in either group intraoperatively. Thus, the study showed that both dexmedetomidine and fentanyl when used with Propofol provide favourable conditions for LMA insertion. Both these drugs cause a fall in the MAP which could be well tolerated in prehydrated, healthy young patients, but may constitute a risk for elderly, hypovolemic and debilitated patients. The incidence of bradycardia is however more with dexmedetomidine and should be kept into consideration while administering this drug. There is also incidence of fall in Respiratory rate which is associated with the patients in which fentanyl is used as an adjuvant compared to dexmedetomidine.

\section{CONCLUSION}

The present study reveals that either dexmedetomidine or fentanyl when used along with propofol provides comparable conditions for the ease of laryngeal mask airway insertion with stable haemodynamic parameters in pre-hydrated, healthy and young patients. However with the use of fentanyl causes mild respiratory depression and cough while the use of dexmedetomidine causes bradycardia without any clinical significance.

\section{REFERENCES}

1. Jerry A. Dorsch, Susan E. Dorsch. Supraglottic Airway Devices. In Understanding anaesthesia equipment. Fifth edition. Philadelphia, USA. LippincottWilliams andWilkins 2008;pg. 461-518.

2. Lamb K, James MF, Janicke PK. LMA for intra-ocular surgery. Effects on intraocular pressure and stress responses. Br J Anaesth 1992;69: 143-47.

3. Yoshino A, Hashimoto Y, Hirashima J et al. Low dose Succinylcholine facilitates airway insertion during Thio-pental anaesthesia. Br J Anaesth 1999;83:279-83.

4. Salem WT. Comparison of midazolam and mini - dose Succinylcholine to aid LMA insertion during Propofol anaesthesia.J Egypt Natl Canc Inst 2000; 12(1): 65-69.

5. Gupta A, Kaur S, Pal Attri J, Saini N. Comparative evaluation of ketamine Propofol, fentanyl - Propofol and butorphanol-Propofol on haemodynamics and laryngeal mask airway insertion conditions Journal of anaesthesia clinical pharmacology 2011;27:74-78.

6. Cheam EWS, Chui PT. Randomised double-blind comparison of fentanyl, mivacurium or placebo to facilitate laryngeal mask airway insertion. Anaesthesia 2000;55:323-326.

7. Driver IK, Wiltshire S, Mills P, Lillywhite N, Howard-Griffin R. Midazolam coinduction and laryngeal mask insertion. Anaesthesia 1996;51:782-784.

8. Zaben K, Qudaisat I, Barazangi B, Badran I: Use of dexmedetomidine as the main anaesthetic agent in patients with laryngomalacia.M.E.J. Anaesthesia 2010;20(4):603-6.

9. Dyck JB, Maze M, Haack, et al. The pharmacokinetics and pharmacodynamics effects of intravenous and intramuscular dexmedetomidine hydrochloride in adult human volunteers. Anaesthesiology 1993;78:813-20.

10. Arian SR, Ebert TJ. The efficacy, side effects and recovery characteristics of dexmedetomidine versus Propofol when used for intraoperative sedation. Anaesth Analg 2002;95:461-6.

11. Turgut N, Turkmen A, Ali $A$ and Altman A. Remifentanyl-Propofol vs Dexmedetomidine-Propofol for supratentorial craniotomy. M.E.J. Anaesth 2009;20(1):63-70.

12. Brain AIJ: The laryngeal mask airway (LMA) insertion manual. Henley, UK: Intavent Research Ltd, 1995.

13. Sivalingham P, Kandasamy R, Madhavan G, Dhakshinamoorthi P. Conditions for Laryngeal Mask Insertion. A comparison of Propofol versus sevoflurane with or without alfentanil. Anaesthesia 1999;54:271-276.

14. Uzumcugil F, Canbay O, Celebi N, Karagoz AH et al. Comparison of Dexmedetomidine-Propofol vs.fentanyl-Propofol for laryngeal mask insertion.European journal of Anaesthesiology 2008;25:675-680.

15. Hanci V, Erdo an G, Okyay RD. Effects of Fentanyl-Lidocaine- Propofol and Dexmedetomidine-Lidocaine- Propofol on Tracheal Intubation Without use of Muscle Relaxant the Kaohsiung J Med Sci 2010;26:244-50.

16. Choudhary J, Prabhudesai A, Datta C. Dexmedetomidine with propofol versus fentanyl with propofol for insertion of Proseal laryngeal mask airway: A randomized, double-blinded clinical trial. J Anaesthesiol Clin Pharmacol 2019;35:368-72.

17. Gupta S, Gadani HN, Shah PR. A comparative evaluation of use of 
dexmedetomidine versus fentanyl for anesthesiainduction with propofol for insertion of laryngeal mask airway. Anaesth Pain \& Intensive Care 2018;22(2):165-173.

18. Ramaswamy AH, Shaikh SI. Comparison of dexmedetomidine-propofol versus fentanyl-propofol for insertion of laryngeal mask airway. J Anaesthesiol Clin Pharmacol 2015;31:21 7-20.

19. Nellore SS, Waychal AD, Rustagi PS.Comparison of DexmedetomidinePropofol versus Fentanyl-Propofol on Insertion Conditions of Proseal Laryngeal Mask Airway [Internet].2016 [Cited October29, 2020];10(11): UC06-UC09.

20. Erkola O, Kortilla K, Aho M et al. Comparison of intramuscular Dexmedetomidine and Midazolam premedication for elective hysterectomy. Anaesth Analg 1994;79:646-53. 